\title{
Chroma Glitch
}

\author{
Data as Style
}

The digital image is too ordered and too rational-and not random enough.

In our experience of it, it lacks... "being."

-JOHN BELTON, "PSYCHOLOGY OF THE PHOTOGRAPHIC, CINEMATIC,

TELEVISUAL, AND DIGITAL IMAGE"

\section{DATA AS STYLE}

Awash in platitudes of progress, transparency, and control, the innovation age also breeds an inversely proportionate dose of fragmentation and failure. ${ }^{1}$ This much was established in the Introduction and chapter 1 . Subsequent chapters have analyzed philosophical and aesthetic strategies for coping with this situation. This chapter builds on the discussion of color as noise in chapters 3 and 4 , extending it to a threefold analysis of the formal attributes of "datamoshing."

Defined as the aesthetic manipulation of digital video compression codecs and decompression algorithms, datamoshing is decked in bold hyper colors and eye-popping animations. It is a subgenre of glitch art, which, as noted in the Introduction, can be found throughout the pop culture, art, and design industries. ${ }^{2}$ Datamoshing's anti-communicative, chunky aesthetic presents a powerful antithesis to the ideology of signal processing, with its unspoken investments in radical compression. As a subgenre of glitch art, datamoshing has been subject to numerous and often disparate definitions. Priscilla Frank defines the genre as a "widespread net art trend in which images crumble into a colorful bath of pixels," a designation that seems to suggest more of a happy accident than anything deliberate or artful. ${ }^{3}$ Alice Pfeiffer, however, writing for the New York Times, defines datamoshing as the "manipulation of compression frames" to produce "an overly pixellated appearance," identifying the crucial role of intentionality in the aesthetic. "Appearance" is the keyword here. Artists-qua-programmers generate excessive pixilation to create a veneer of chaos and lack of control. This 


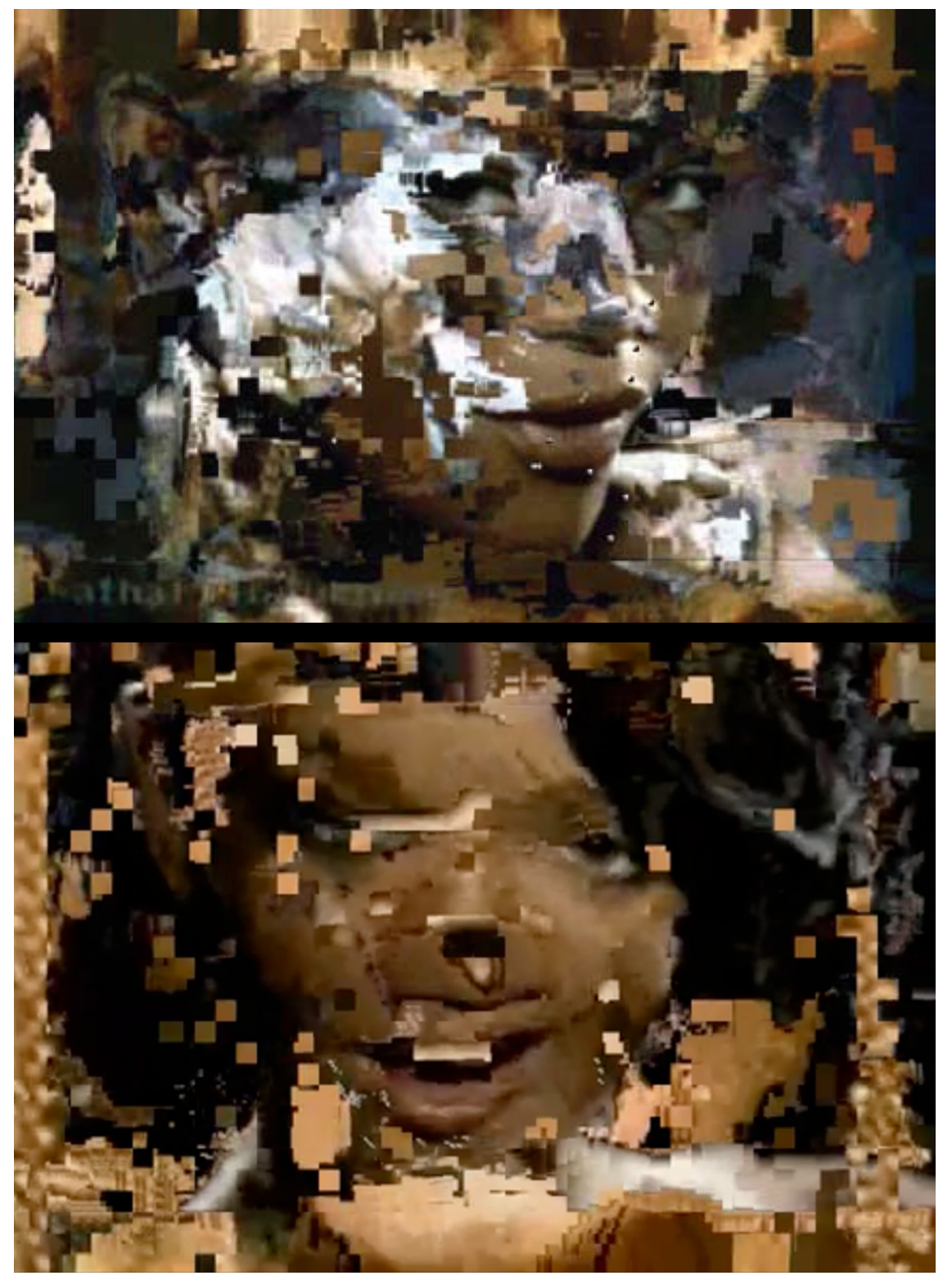

FIgURE 22. Paul B. Davis and Jacob Ciocci, Compression Study\#1 (Untitled Data Mashup) (2007), digital video, color, 2 minutes. This pioneering datamosh mixes Rihanna's smash hit Umbrella (2007) with the Cranberries' Zombie (1994). 


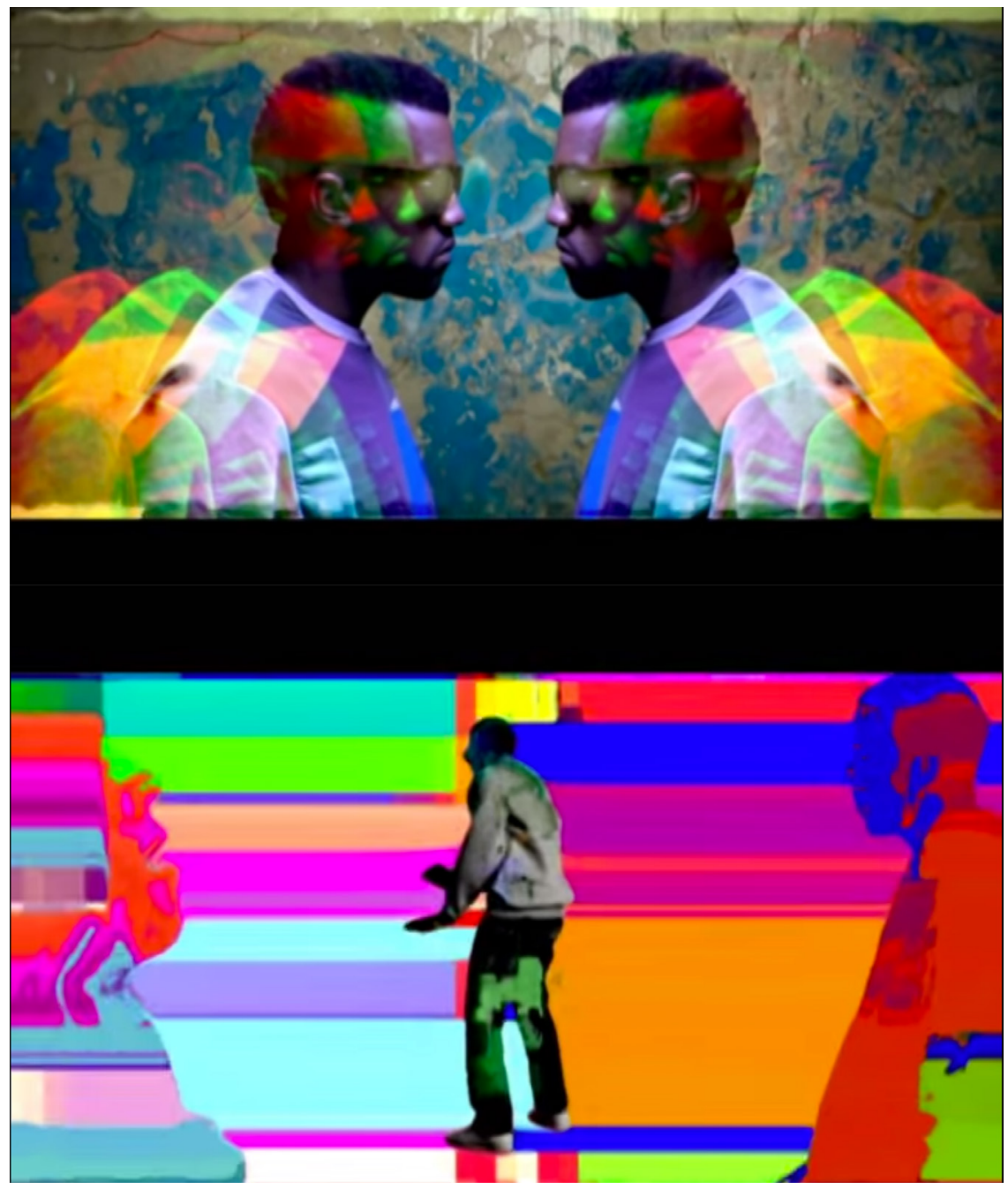

FIGURE 23. Kanye West. Welcome to Heartbreak (2009). Directed by Nabil Elderkin, edited by Ryan Bartley at Ghost Town Media. Digital video, color, 4 minutes, 23 seconds.

out-of-control façade is definitive of the genre, one that first and foremost involves a controlled and strategic manipulation of digital media.

One pioneering example is Paul Davis and Jacob Ciocci's Compression Study \#1 (2007) for which they combined a clip from Rihanna's smash hit video Umbrella (2007) with a clip from the Cranberries' music video Zombie (1994), featuring vocalist Dolores O'Riordan, in addition to a selection of television clips and internet videos. The piece begins with an image of Rihanna singing which, a few seconds 
later, begins to break apart. Pixels formerly concealed in shimmering bronze flesh break into macroblocks, bleeding until the entirety of the image has morphed into an image of O'Riordan singing in the Cranberries' Zombie. The effect is both sudden and poetic, designed to mosh the image and sound simultaneously.

Similar examples of datamoshing in mass media and pop culture music videos include Chairlift's "Evident Utensil" (2008); Linkin Park's "New Divide" (2009); Kanye West's Welcome to Heartbreak (2009), directed by Nabil Elderkin and edited by Ryan Bartley at Ghost Town Media; and A \$AP Mob’s "Yamborghini High" (2016), edited and directed by Shomi Patwary of Illusive Media. Whether off-line or online, datamoshing draws from emerging trends in internet culture and the deliberate deterioration of high-resolution images into "colorful baths of pixels," as Frank puts it. ${ }^{5}$

The "moshing" of computer code marks one significant difference between glitch art (and by extension, datamoshing) and the more general forms of visual or conceptual noise found in the work of John Baldessari (Introduction), Ryan Trecartin (chapter 3), and Thomas Ruff (chapter 4). Where glitch art and datamoshing involve hacks into computer algorithms, they are unlike other techniques in visual noise, which could include anything from abstraction to loosely defined anti-communicative breaks from representational norms. At the same time, as a subgenre of glitch art, datamoshing embodies a slightly narrower definition than the more general category of glitch art. For example, the latter includes work from Rosa Menkman, Jon Cates, and Andrew Benson, which certainly involve programming-based interventions into computer processing, but is not to be confused with datamoshing because a good deal of their work does not involve specific interventions with video compression codecs (I provide a technical exegesis of video compression codecs below).

Minor genre distinctions aside, it is undeniable that the chunky digital artifacts of datamoshing and glitch art seem to say nothing and communicate even less. How then can we explain their popularity as a cultural style, from fine art to mass media? How did these formerly erroneous glitches manage to move from signifying an unwanted state to the essence of a pop culture fashion, whether as nightclub special effect or hip décor for Bloomberg Businessweek? ${ }^{6}$ What is it about glitch art and datamoshing that provides added value, whether organic, trendy, superficial, counterhegemonic, or otherwise?

This chapter provides a preliminary set of responses by analyzing key examples of datamoshing in a threefold framework of the analog and the digital. I do not have in mind the physical technologies we are familiar with, but rather the analog and digital as metaphors; as aesthetic strategies connected to specific historical and material registers. My first conceptual framework for the analog and digital adopts the lens of colorism, which denotes the particular way an artist or designer uses color. For instance, saturated complementary colors typify the fauvist style, while strong blacks denote German expressionism, and primary hues embody cubism or 


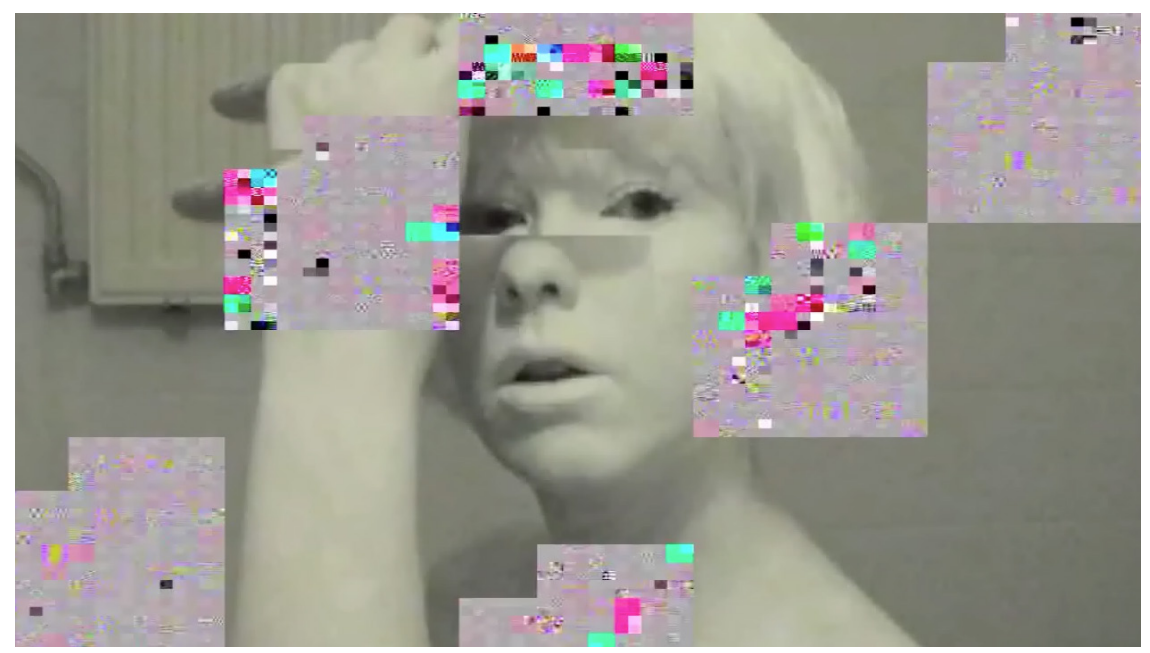

FIGURE 24. Rosa Menkman, Demolish the eerie u25bcoid (2010). Digital video, color, 1 minute. Menkman's work masterfully illustrates data as noise, while still conveying deeper meanings. Courtesy of Rosa Menkman.

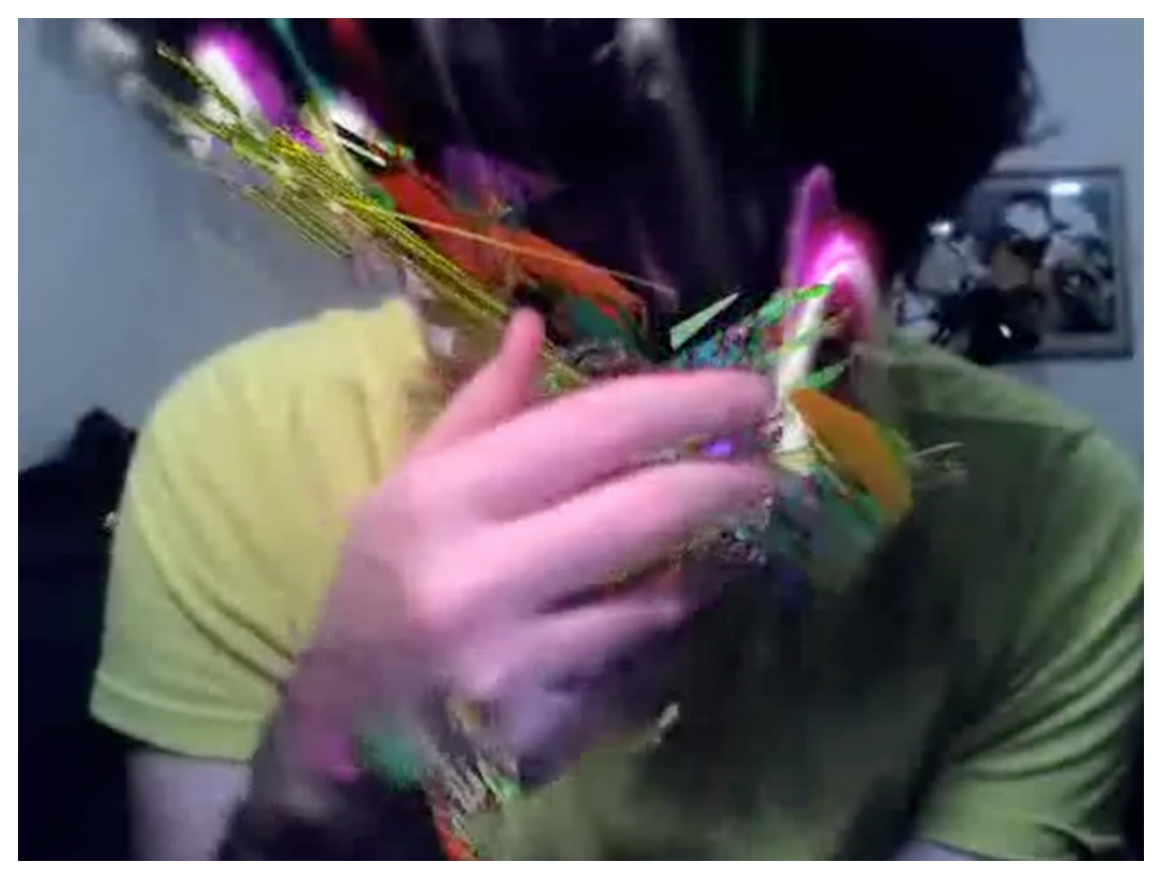

FIGURE 25. Andrew Benson. Sparkle Face Test (2011). Digital video, color, 40 seconds. Made with Max SP, a motion-tracking software, Benson uses his hand gestures to control the multicolored rays of glitch abstraction. Courtesy of Andrew Benson. 
De Stijl. 7 My second framework considers compositional structure. I draw on Gilles Deleuze's theory of aesthetics, articulated in terms of analog and digital synthesizers. The third frame turns to Heinrich Wölfflin's account of the historical development of style from the High Renaissance to the baroque. The chapter concludes that the analog and digital, as metaphors removed from the technical systems they normatively signify, provide insight into the development of an aesthetic paradigm rooted in uncertainty, hyperactivity, and sensory overload; not coincidentally, the very same attributes definitive of the contemporary media environment.

\section{COMPRESSION ECONOMIES}

To be clear, the popularity of glitch aesthetics does not imply that the long-standing demand for media fidelity and crisp visual imagery has dissipated. To the contrary, the vast majority of consumers, producers, and users expect as much, especially from well-advertised "new media" products like HDTV, IMAX cinema, or Blu-ray Disc, all of which turn on the successful engineering of compression algorithms for digital formats. Such industry standards derive from a broader history of engineering, beginning with nineteenth-century research in psychophysics, through acoustical engineering conducted at research centers like Bell Telephone Laboratories, Xerox Parc in the mid twentieth century, and Tesla and Google Labs today. ${ }^{8}$ In short, decisions for establishing which codecs to use in optimizing a signal are based on rigorous research into communicative efficiency and control.

A host of media theorists have critiqued our culture's relentless drive for smaller and more compact media, capable of delivering cleaner, faster, and more enriched content. ${ }^{9}$ Vernacular digital formats like the GIF, $\mathrm{MP}_{3}, \mathrm{MP}_{4}, \mathrm{JPEG}, \mathrm{PDF}, \mathrm{PNG}$, TIFF, or TARGA all have histories of radical data compression, straddling tensions between innovation and experimentation, on the one hand, and the need to comply with engineering standards, on the other. In order to become an industry standard at all, compression schemes must be approved by the International Organization for Standardization, an international standard-setting body for the internet, and the International Telecommunications Union (ITU), established in 1865 as the International Telegraph Union. According to media historian Sean Cubitt, the ITU is the "oldest intergovernmental treaty body still functioning today." Its tasks include safeguarding the "interoperability of telecom systems, pricing regimes for international calling, and infrastructures for audiovisual services," which includes "moving image encoding," or simply, "low bit-rate communications." ${ }^{\circ}$ One result of this governance is an endless stream of faster and more efficient "universal" standards for global communication media. As consumers, we have been trained to expect this much, and more, at ever cheaper prices.

When new and improved technologies fail to deliver these cheaper and faster options, or reveal a brief stutter, dropped signal, or failure to load, one is immediately disappointed. What happened to all of these rigorous standards? We are 
more lenient with internet imagery, where the occasional glitch is tolerated, but too much interruption, static, or noise yields the same dissatisfied results, causing us to revert back to older media-the telephone, face-to-face visitsinquiring what "went wrong." In sum, global culture's hegemonic imperatives for technocratic progress ensure that innovation in compression engineering will continue to be big business in years to come. But consider now that the very thing that is unwanted in prosaic media experience is precisely what is sought after in glitch art. To better understand these seemingly unwanted glitches, we must set aside these otherwise normative cultural demands for high resolution, fidelity, and verisimilitude.

\section{THREE APPROACHES TO STYLE}

\section{Analog Color versus Digital Colors}

While my goal here is to depart from literal digital and analog systems, a cursory technical distinction is nonetheless in order. The analog is defined as a series of continuous wave forms and infinite gradations of data, as found on a vinyl record, in the sound waves exchanged in face-to-face conversation, or in analog electronic computers. Data operate through analogy. An analog computer, for example, takes a quantity from a physical source, like an electric current or sound, and abstracts it into a corresponding value that is directly representative of the input, such as a sound wave or X-ray. Data are then transferred from input to output in a continuous form, which a producer can control by "modulating" the single frequency between them.

Digital technology, on the other hand, is defined by a series of discrete units of information from which other formations can then derive. In a digital system, such as a digital computer, the basic language is arithmetic. A digital computer operates through a rigorous quantization of discrete numerical values, often in binary form, where each unit (o or 1 ) is the baseline from which all other digital media derive. But digital technology is not exclusive to modern computing. Our two sets of five digits known as fingers are a digital system that has always been a part of human culture.

James Small has defined the difference between the analog and the digital by comparing them with the slide rule and the abacus, respectively:

In the abacus, quantities are represented by a number of beads, thus the quantity being represented can only vary, up or down, by a minimum of one bead-there are no partial beads.... all operations are performed as a series of additions or subtractions. In contrast, the slide rule represents quantities as continuously varying magnitudes: in this case length. The granularity of the result is limited only by the coarseness of the scale used to perform the measurement. ${ }^{11}$

The British artist and color theorist David Batchelor makes a series of insightful claims about colorism in contemporary art based on the distinction between 
TABLE 1. Analog and Digital as Cultural Technologies

\section{Analog Technology}

- Analog technology is identified by its continuous and uninterrupted waveforms. Examples include vinyl records, audiocassette tapes, "wet" photography, and the human voice.

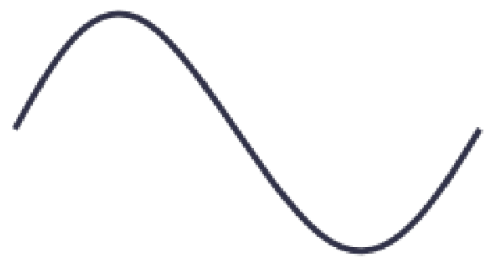

\section{ANALOG SOUND WAVE}

- If an "analogy" implies a general likeness or indexical relation between two things or concepts, analog technology likewise involves an equivalence between two sets of terms. With traditional "wet" photography, for instance, light is captured on a photosensitive substrate which, after processing in the darkroom, reveals an analogous imprint.

\section{Digital Technology}

- Digital technology is defined by a series of discrete units of information. For example, binary digits consist of two integers, 0 and 1 , which are the basic units of measure used to calculate and build all additional developments.

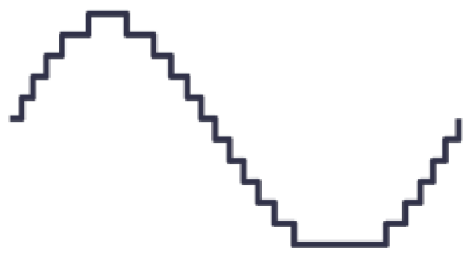

\section{DIGITAL SOUND WAVE}

- Digital systems are not exclusive to computing, though this is where they are most commonly affiliated. Digital technologies also include the five digits on each hand, an abacus, or any other system where individual units are divided into discrete entities.

analog and digital. I ultimately take issue with his periodization of color styles, but they are worth exploring as a preamble to his more germane conceptual distinction between analog color and digital colors.

The widespread introduction of industrial-ordered color after World War II, Batchelor argues, led to an overall digitization of color, by which he has in mind a style, not a literal technology. ${ }^{12}$ "Colour-chart colours" contributed to a "change in the use and understanding of colour. This might be called the digitalization of colour, whose opposite is analogical colour." ${ }^{13}$ A number of artistic and industrial design examples support this claim, but many examples also contradict it. Table 2 provides an overview of the comparison between the analog and the digital as styles of color and composition.

In Batchelor's theoretical approach to the twin concepts, analog color denotes "the one" and digital colors connote "the many." As a singular phenomenon, analog color has no inner or outer divisions, formal limits, or intrinsic distinctions. It is conceptually and perceptually undifferentiated, regardless of discipline or medium. Analog color invokes the "magical splendor" of witnessing color phenomena mixing and melting in the sky, a moving image or a spinning wheel. Where analog color is a wheel, digital colors are the "infinite and infinitely thin 
TABLE 2. Analog and Digital as Stylistic Modes

\begin{tabular}{|c|c|c|}
\hline $\begin{array}{l}\text { Colorism (color } \\
\text { treatment) }\end{array}$ & $\begin{array}{l}\text { Analog Color } \\
\text { - Continuous, undifferentiated color; } \\
\text { no inside or outside; indivisible } \\
\text { and indecipherable mixtures. Color } \\
\text { "spreads flows bleeds stains floods } \\
\text { soaks seeps merges. It does not } \\
\text { segment or subdivide" (Batchelor, } \\
\text { Chromophobia, 86). } \\
\text { - Gamut is often represented as } \\
\text { a circle, allowing mixing and } \\
\text { blending. } \\
\text { - Nietzsche's Dionysian chaos } \\
\text { - Associations with romanticism, } \\
\text { utopia, childlike innocence. } \\
\text { Artistic illustration in Seurat, } \\
\text { Rothko, Rist. }\end{array}$ & $\begin{array}{l}\text { - Gamut is represented as a chart or } \\
\text { table with distinct divisions between } \\
\text { colors. } \\
\text { - Nietzsche's Apollonian individuation } \\
\text { - Associations with nominalization, } \\
\text { order, systems, and hierarchies. } \\
\text { - Artistic illustration in Mondrian, Riley, } \\
\text { Hirst, Bulloch. }\end{array}$ \\
\hline $\begin{array}{l}\text { Compositional } \\
\text { Structure }\end{array}$ & $\begin{array}{l}\text { Analog Synthesizer } \\
\text { - Analogical compositions are } \\
\text { "modular," involving fusions of } \\
\text { difference. } \\
\text { - Color is used to structure an } \\
\text { analogic and continuous language. } \\
\text { - Relation to the haptic; the "manual } \\
\text { lines" of Jackson Pollock, having } \\
\text { neither inside nor outside; an } \\
\text { abysmal chaos deployed to the } \\
\text { extreme. } \\
\text { - Generative of a new creative order } \\
\text { and rhythm to come, emerging } \\
\text { from the ground of (haptic) } \\
\text { sensation. }\end{array}$ & $\begin{array}{l}\text { (Digital) Code } \\
\text { - Less a diagram than a symbolic code. } \\
\text { - Units are grouped visually in terms of } \\
\text { binary opposition. } \\
\text { - Colors are derivative of predetermined } \\
\text { codes or machinic orders. } \\
\text { - Piet Mondrian's "digital grids" create } \\
\text { a "code" that is cerebral and lacks } \\
\text { sensation. }\end{array}$ \\
\hline
\end{tabular}

spokes inserted in the wheel." ${ }^{14}$ Consider the effect of watching a spinning wheel, even a bicycle wheel, where the individual spokes (or colors) become indecipherable. In rapid movement, we do not experience one color in isolation but instead, an ineffable mixing of all the colors on the wheel, as a whole. In this way, analog color touches on holistic visions of a mystical cosmos, like Heraclitus's "All things are one," ${ }^{15}$ or Spinoza's "univocity of being" as interpreted by Gilles Deleuze. One could also align analog color with Galloway's recent work on François Laruelle, or Nietzsche’s Dionysian "primal oneness," where analog color, like some conceptions of noise, act as a signifier of undifferentiated beings.

In the history of art and design, analog color can be identified in the amorphous, shape-shifting colors of an Olafur Eliasson or James Turrell installation; the melting, multilayered hues of a Marilyn Minter photograph; the soft and melting 
pigments in Terrence Malick's Days of Heaven (shot with cinematographer Néstor Almendros); or one of Mark Rothko's meditative color fields. To propose Ryan Trecartin's work as an example of analog color may be a stretch, though his strategies do involve the same kind of categorical blurring required for analog color's anti-individualism and lack of difference. And hence, in these pre- and postwar examples of analog colorism, we find an active mode of color use that is not specific to medium or exclusive to postwar periodization, as Batchelor indicates. ${ }^{16}$

To take this a step further, analog color-as concept-can retroactively be understood to play a vital role in the history of science and technology. Newton's optical experiments of 1666 , for example, were initially analog. In his most famous experiment, also observed by Batchelor, Newton made a small hole about $1 \mathrm{~cm}$ in diameter in a window shutter in a dark room. He then placed a prism in front of the small hole, the only point where outside light could enter the otherwise black box. In studying the incoming daylight refracted through the prism, he observed what has become known as the seven spectral hues, which he at first referred to as a "kaleidoscopic promiscuity." ${ }^{17}$ His choice of words captures the essence of ana$\log$ color as an ineffable and mysterious mixture of scintillating visual displays, akin to the ephemeral noise framing the background of experience observed by Nietzsche, Attali, Kittler, and Virilio, as noted in the Introduction. At first, then, Newton's perceptual experience was analogic and continuous. He observed white light as "a confused aggregate" of colored rays. ${ }^{18}$ Shortly thereafter, when he classified and divided these colors into seven discrete identities (red, orange, yellow, green, blue, indigo, and violet), he catapulted them into the digital terrain. ${ }^{19}$ The distinction is key because it elucidates the difference between phenomenological experiences of color on the one hand (i.e., holistic, analogic, continuous, and, typically, poetic and romantic), and techno-rational models of classifying and controlling color on the other. And thus we arrive at digital colors. ${ }^{20}$

One must be careful not to be swept away into the poetic idealizations of analogic color. We cannot lose sight of the fact that, in order to use color at all, it is essential to create standards, so that multiple persons can use the same color with a shared understanding of the differences between it and other colors. In short, in order to use color in any practical or collective way, it is necessary to turn analog color into digital colors. CIE lab, HTML, Pantone, the 12-hue color circle, and hexadecimal color charts are all examples of digital color systems inasmuch as they all systematically divide color along a continuum, grid, matrix, chart, or index. Digital colors retain distinctions between inside and outside: where one color begins and where it ends, and what one color is and what it is not. Zoning, distinction, and separation are the catchwords of digital colors. If analog color is Dionysian in its maddening blurs, digital colors are Apollonian in their levelheaded individuation. ${ }^{21}$ Artistic examples abound in modern architecture and design, which unequivocally privileges line, form, and order over shape-shifting color. In art, examples of digital colorism can be found in Robert Rauschenberg's 
black and white monochromes, Gerhard Richter's color chart paintings, Warhol's aggressive and jarring separations between screen-printed color and line, Peter Halley's cells and conduits, and most of Piet Mondrian's work. All of these artists engage digital colorism in unique ways, but not all of them use electronic digital computers to do so, again illustrating how analog color and digital colors are here construed as styles, not specific to medium or platform. ${ }^{22}$

To sum up thus far, the two modes of working with color-the analog and digital-are radically distinct but nonetheless play a pivotal role in producing aesthetic value and signified content. How and why an artist or designer makes certain choices with color is deeply meaningful, as is their treatment of compositional space.

\section{The Analog and Digital as Compositional Logic}

My second analytic framework adopts the lens of compositional structure. Key fragments selected from the late work of Gilles Deleuze, when braided back together, provide an account of his aesthetic theory of composition (see Table 2). In these fragments, Deleuze addresses cinema, painting, and color, theorized through analog and digital metaphors. ${ }^{23}$ His initial approach follows Goethe's pivotal 1810 Zur Farbenlehre (Theory of Colours), where sensation is placed prior to interpretation and cognitive recognition. ${ }^{24}$ Goethe's approach was in itself eccentric, radically reversing not only Newton's claims about color but also, art history's long-standing opposition between colore and disegno, which privileges the role of design, line, and structure over sensuous color. As can be expected, Goethe's theory of color only serves Deleuze as a starting point, after which he moves in an orthogonal direction. ${ }^{25}$ While Deleuze's initial loyalty to Goethe lands him on the romantic side of analog style - versus the discrete logic of digital code-his recourse to synthesizers in articulating his theory, and his invocation of the "diagram," complicates any clear-cut classification. To reiterate, the following discussion of analog and digital synthesizers presents metaphors for creative compositional structures, not descriptions of physical media.

The creative process never begins with a blank slate, Deleuze argues; rather, an artist begins with a whole history of art and cultural clichés already written on the canvas or screen. The painter "has many things in his head, or around him, or in his studio ... [and] everything he has in his head or around him is already in the canvas . . . before he begins his work." ${ }^{26}$ Noise and color are implicit on a seemingly empty white canvas, as Rauschenberg's white monochromes suggest (see chapter 2). Before beginning a piece, one is required to intervene and clear the noisy clichés. This initial creative gesture is therefore destructive, it must unhinge, mobilize, and "deterritorialize" convention. Scraping, scratching, and clawing are all valid techniques for accomplishing this. From here, there are two possibilities for the composition: the analog and the digital.

The first kind of compositional structure is based on the analog synthesizer. This kind of composition is created by analogy, which is to say, using heterogeneous bits 
and fragments-scraps and leftovers from the destruction process-to establish an immediate or qualitatively new connection between them. If used successfully, one creates a diagram. In his theory of cinema, Deleuze identifies the need for freefloating, subjective affect (noise, color-as-color, abstract pixels) to be modulated through a synthesizer. Controlling such free-floating modulations allows one to construct a "diagram," a compositional system created from re-ordered units of raw sensation. Paul Cézanne, Deleuze notes, used basic geometry to develop analogies between line and color, elevating these relations into his own diagram, expressive of movement and unique sensation. Antonin Artaud's "signs, groans, whispers, [and] moans," and Francis Bacon's melting, torn, disfigured bodies also used raw sensation to reorder them in depicting "flesh versus meat"; a diagram of new color sensation versus clichés already tired and dead. ${ }^{27}$ Destruction must occur, producing raw color affects, but it is then the job of the artist to re-order and control them. Noise, like color, "allow[s] itself to be heard" through specific modulations between order and disorder, like a "sieve whose mesh will transmute from point to point." ${ }^{28}$

Insofar as color / noise cannot be modulated, it sustains "hysteria." This is not yet a diagram, but it is the raw material for making one. In the case of Jackson Pollock, Deleuze argues, we find an artist who successfully clears the ground of cliché and convention, but then pushes his "manual lines" too far, producing only abysmal chaos with no inside or outside, failing to generate a synthesizer or active filtering mechanism to modulate color effects into a new rhythm and order that would form a diagram. ${ }^{29}$

If analog modulation deploys color affects to synthesize and in-form matter, color in digital code can only be applied to predetermined units in an a priori structure. Deleuze's notion of digital code is largely akin to "digital colorism." Both involve discrete systems of pre-divided units (individual colors or sections of a grid) for processing and determining in advance all future possible variations. To adopt digital code as the guiding rubric in a composition means generating only predetermined relations, a "limited set of simple, discrete forms" that are less diagrams or active machines than "symbolic codes." ${ }^{\circ 0}$ Mondrian's compositions are such examples of digital code; his choices are always binary and cerebral, "lack[ing] sensation," but not so much that he eclipses spontaneity entirely. Mondrian does not reduce the entire composition to this code, Deleuze insists, he leaves open a few unpredictable steps for new moments and mixtures. ${ }^{31}$ Like the abacus described above, the digital composition requires that all operations be "performed as a series of additions or subtractions" of the initial code. ${ }^{32}$ This is why the digital synthesizer does not bear the same kind of spontaneous or creative capacity as the analog synthesizer, but is instead referred to by Deleuze as a kind of pre-sanctioned series or reiterations of what already exists. If we translate this model back into the datamoshing, we can reexamine issues of creative control as pivotal in determining the relative value of a work in the genre. To do this, a brief technical exegesis of digital video compression is first in order. 


\section{Digital Video Compression}

A digital video frame is composed of mathematical codes and functions known as compression schemes or codecs, responsible for the transmission, storage, and distribution of information. Relative to the still image formats designed for the Internet or related media-GIF, JPEG, TIFF, TARGA, or PNG-digital video is one of the most challenging to compress because video files tend to be larger and informationally denser, due to their time-based nature, and demand to be seen at higher resolutions (HDTV). Because superfluous and repetitive information must be eliminated to keep the file size down, digital video compression codecs tend to rely on semi-autonomous modules known as "macroblocks," units of $4 \times 4$ or 16 $\times 16$ pixels of video data, grouped together and averaged for their hue, saturation, and brightness values. Macroblocks are the chunky pixelated blocks that appear on screen, generally misrecognized as pixels. But these sequences are not individual pixels, they are groupings of similar bits of data, based on luminance and chrominance values. Macroblocks are not unique to video; they are also common in JPEG, H.261, and various MPEG formats, all of which use lossy compression. ${ }^{33}$

As noted in chapter 4, most digital video uses lossy compression. The lossy compression scheme reduces bits of data in a file by identifying unnecessary information and removing it, making it possible to distribute the image or sound files faster and more efficiently. The trade-off is that lossy compression produces a file with a lower quality than the original photograph, video, or sound material. Nonetheless, lossy compression formats like the $\mathrm{MP}_{3}$, GIF, or JPEG are ubiquitous, due to increasing demands for faster and more frequent transmission, coupled with a diminishing concern for image quality (at least on the internet).

The compression algorithms used to engineer these lossy formats further divide data into three different frame types: I-frame, P-frame, and B-frame. The I-frame (intra-coded picture) or reference key frame is the first frame inserted and used to indicate a significant change in content, like a change in scenery or sudden movement. ${ }^{34}$ It is in a sense the most important key frame, the least compressed, and does not rely on other picture frames to decode its data. Contrarily, P-frames, "forward predicted frames," and B-frames, "bidirectional frames," are more compressed, used to maintain consistency in movement and data. The I-P-B-frame logic derives from the classic film animation technique known as interpolation, a technique that assumes that a patch of color will remain consistent for at least a few frames in a sequence, so redundant or repetitive data can be discarded. Interpolation is common in MPEG codecs, especially with the H.261 codec, underlying YouTube's proprietary .flv format. ${ }^{35}$ One result of this compression scheme is a dramatic reduction in information relative to the original, which means much less color detail and nuance, but results that are nonetheless "economically satisfying," as Sean Cubitt puts it, at least for those who value smooth and speedy downloading over detail and subtlety. ${ }^{36}$ 
Because the I-frames are the most expensive frames-containing more data and instructions regarding movement, the most logical way to reduce overall file size is either to minimize movement in a video image from the start or to develop more sophisticated vector-prediction algorithms to eliminate movement perceived to be unnecessary. For Cubitt, this has led to a "self-fulfilled prophecy of YouTube videos" populated by immobile, "talking heads" and minimally changing animations. ${ }^{37}$ Datamoshers exploit this logic by deleting key I-frames, the necessary signposts to signal a dramatic shift in the clip. What remains are only the "filler" P- and B-frames, which contain motion vectors that only note differences in the position of the macroblocks between the immediately surrounding frames..$^{38}$ As a result, the rendering algorithm does not understand how or when to properly shift to accommodate changes in content, so it simply moshes the content together, creating the liquid but chunky block effect definitive of the genre. The extent to which an artist has control over the I-frame deletions determines the potential for excellence in a work.

To be clear, all creative work should be controlled. Even techniques in chance and randomness require an artist to make explicit choices as to where and how a piece will begin and end. Artists should be able to master their tools and fluidly negotiate between chaos, accident, and chance, on the one hand, and a consciously desired outcome, on the other. The Los Angeles-based media artist Andrew Benson's Sparkle Face Test (2011) is an excellent example of such interaction between chaos and control. And while this work is not a datamosh in the strict sense I have defined, the piece offers a unique use of vector-based graphics to create a glitch aesthetic in time-based media. In this exuberant 40-second digital video, Benson programs Max SP, an interactive motion-tracking software to create the illusion that he is sculpting multicolored abstractions with his hands. The piece is tightly choreographed, leaving little room to misinterpret it as anything but extremely well measured (i.e., his modulated diagram), and yet, the communicated meaning is deliberately and precisely the opposite: a seemingly chaotic, "colorful bath of pixels" swimming in uncontrollable arrays. The tension between chaos and control is the wellspring from which his diagram is drawn.

In fact, the issue of control is so central to datamoshing, it may even be more vital than the pixelated "errors" that define its façade. Control over technological error is not only datamoshing's form of appearance, it is also its condition of possibility. Understanding the backend technical decisions for Compression Study \#1 (fig. 22), helps us to see its value as an early datamosh (2007). If the control process is to include both the hack and the sculpting of the final work (i.e.: the diagram), then a problem emerges midway through Compression Study. In the first RihannaCranberries sequence of the piece, the artists' results were achieved by elegantly mixing two video sources together, resulting in the I-frames of one image appearing to pause on screen and then suddenly vaporize into the next image. ${ }^{39}$ They also chose two music tracks whose melodies synchronized to achieve this fluid 


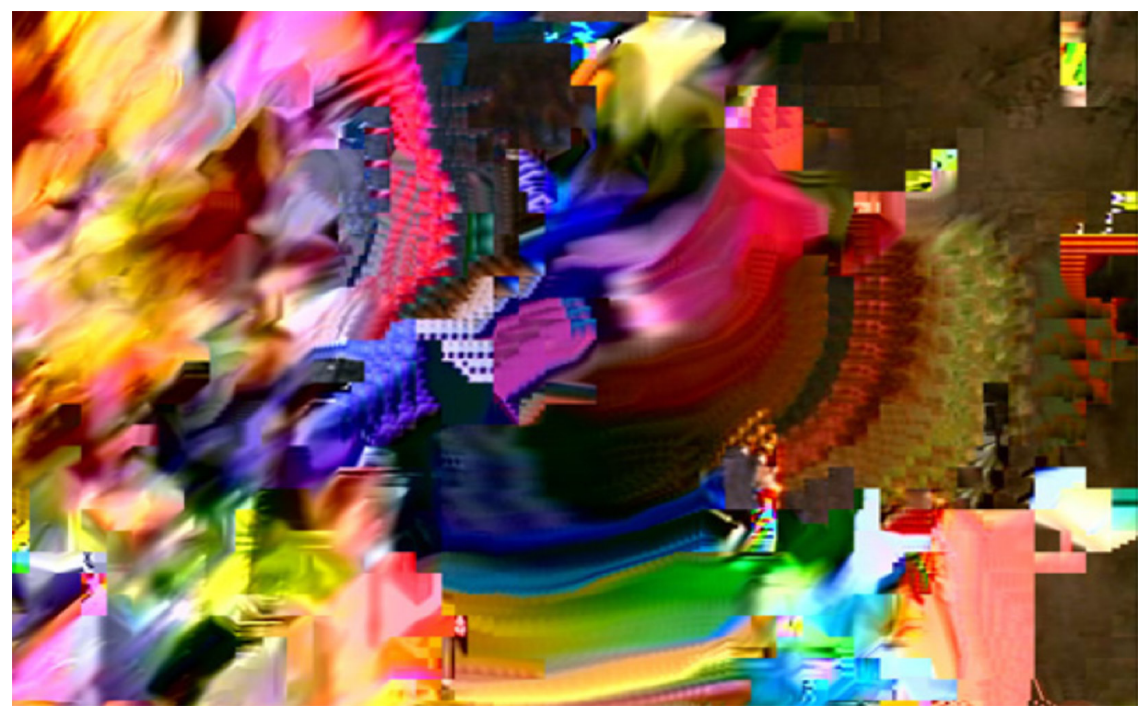

FIGURE 26. Takeshi Murata. Monster Movie (2005). Digital video, color, 4 minutes, 30 seconds. An accidental error transformed into an elegant datamosh.

yet harmonized affect when mashed together in the remix. Because the sound is so evenly integrated, the pixels, while choppy, seem to fuse a little more smoothly. However, if a successful diagram requires control over the entire composition, then this mandate has only been achieved for the first two scenes, after which the careful balance between chaos and control dissolves and the rhythm is lost. Their diagram flops as the piece segues into a series of retrogressive montage cuts of children's television shows. The diagram is botched.

I end this chapter with a final example of a pioneering datamosh where the diagram is maintained throughout. Japanese American media artist Takeshi Murata's four-minute Monster Movie (2005), made two years prior to Compression Study \#1, was developed from a "wild" error Murata encountered while downloading a movie file. He then isolated and repeated the error numerous times, creating this elaborate work, which dances between the analog and the digital.

Monster Movie opens with an amorphous mass of gray pixels, somewhat resembling the shape of a head rising from a pool of what appears to be ice water in an underground cave (the image is taken from the 1981 film Caveman). But as soon as the filmic image reveals portions of the monster's head, hair, and fangs, it disappears back into the soupy mix of monochromatic pixels. Edgy, pixilated colors undulate and flow in wave formations, smoothly animating the same monster head to ever so briefly reemerge, only to sink back down, again and again. Colors detach from the familiar, cognizable world, as in Bacon's paintings, where forms morph, merge, and bleed into each other through color mixing. But here the technique 


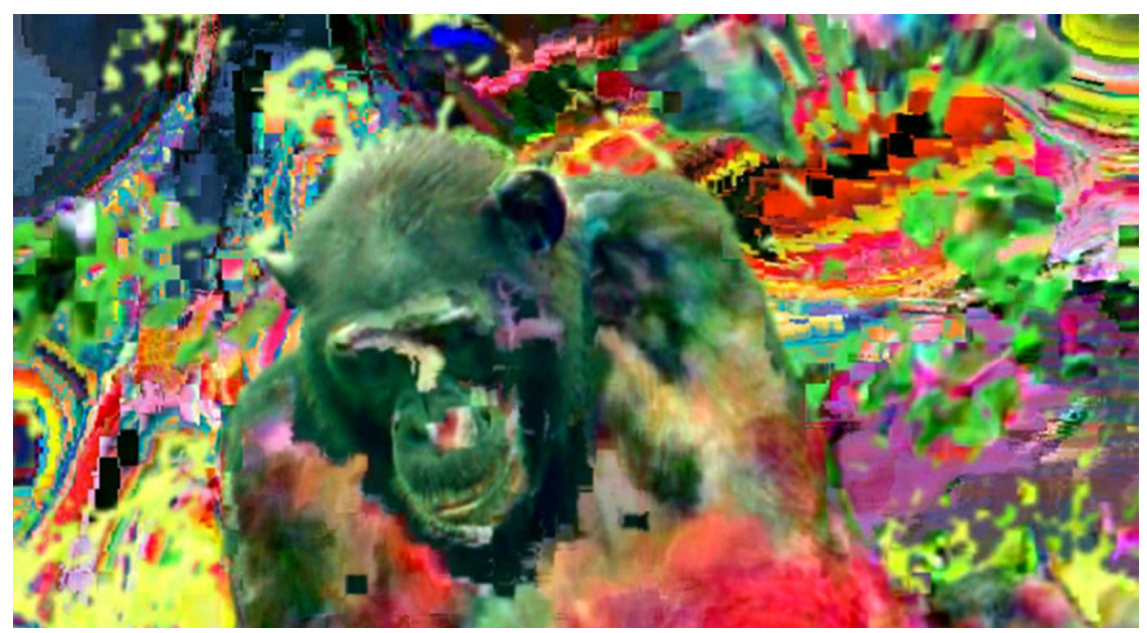

FIGURE 27. Takeshi Murata. Monster Movie (2005). A "catastrophe of experience" (Deleuze, Francis Bacon: The Logic of Sensation, xix).

is more aggressive. Backgrounds fuse with foregrounds as foregrounds transcend themselves a hundred times over. The image's focal point counterintuitively generates infinite space and depth from within itself, destroying conventional notions of horizon, ground, or perspective. With no stable focal point, Monster Movie clears clichés of visual representation..$^{\circ}$

After this clearing, a "catastrophe" emerges from which, in contrast to the Kantian sublime (discussed in chapters 6 and 7 ) reason does not achieve recuperation. Daniel Smith explains Deleuze's orthogonal interpretation of Kant's aesthetic sublime in appropriately colorful language: "My entire structure of perception ... is in the process of exploding.... I can no longer apprehend the successive parts, I cannot reproduce the preceding parts as the following one arrives. ... I can no longer recognize what the thing is. I can no longer qualify the object in general." ${ }^{41}$ It is in this state of "incompossibility" that a clear-cut distinction can be made between Deleuze's aesthetic philosophy of the sublime on the one hand, and Kant's more traditional one, on the other. ${ }^{42}$ For Kant, as I address in chapters 6 and 7, the aesthetic sublime denotes a dizzying state in which a subject is suspended in awe, but, and this is key, this catastrophic encounter introduces only a temporary gap in cognitive experience, from which the subject is ultimately rescued by the faculty of reason. ${ }^{43}$ For Kant, the aesthetic sublime is merely a demonstration of the force and magnitude of reason to deal with situations that at first appear beyond it. As he puts it in the Critique of Judgment, "aesthetic judgment refers not merely, as a judgment of taste, to the beautiful, but also, as springing from a higher intellectual feeling, to the sublime." ${ }^{44}$ In classical aesthetics, reason, and hence understanding, always trump the raw and deceptive realm of sensory affect (color, noise, glitch). 
By contrast, for Deleuze, a subject voluntarily and intentionally enters this state, at first resembling a kind of (Freudian) trauma in experience. But, in opposition to Freud's normative trajectory of therapeutic resolve (and Kant's recuperation by the force of reason), Deleuze lingers in the hysteria..$^{45}$ The successful artist must land in a "purely aesthetic clinic" for hysterics and schizophrenics, he writes, where the noisy stopping and stuttering of chaos and chromatic profusion can be sustained long enough to transform black and white into an aesthetic more "related to green and red." 46 The multitude of colors, with avid juxtapositions and ceaseless contrasts of hue, allow visual noise to proliferate to an extreme, suggesting a lack of control and pure chaos akin to Jackson Pollock's lines or datamoshing's trademark visual signifiers. The power of color is movement from singular, linear, and rational frameworks into noise, nondistinction, and the deceptive but vital qualities of visual perception.

One cannot leave their colors or composition in this state, however, at least not if one wishes to make "art." The next step is to modulate the diagram or code. This returns us to the controlled catastrophe of Monster Movie. Accordingly, Murata instigates this crisis in knowledge and perception, but does not allow it to overtake the work. Rather, chaotic color is modulated to persist at his discretion and, through repetitive animations and editing, he establishes a new rhythmic order. Just as the moshed artifacts reach maximal color intensity and abstraction, they settle into a holding pattern, echoed through the half-identifiable yelps and groans heard throughout, mixed with the offbeat percussion track by Plate Tectonics. So while Murata modulates and develops errors, he also transforms them, bringing them back into the domain of aesthetic control. This is his diagram: a rhythmical composition balancing the tension between the jagged and rigid pixelated artifacts of the digital, on one hand, and their elegant, continuous animations, on the other.

A similar strategy is used in Murata's Untitled (Pink Dot) (2007), where pixelated digital video artifacts are fused with images of Sylvester Stallone from the 1982 film Rambo: First Blood. Throughout the piece, a large, pulsating fuchsia dot gently flickers off and on in the screen's center. The visual motif resembles glitches and noise effects in such classic video artworks as Steina and Woody Vasulka's Noisefields (1972) or Scott Bartlett's Off /On (1972), where the core pulse and rhythm of the work breaks with visual convention and depth perspective, reframing a noise-laden visual experience of chromatic, synthetic pulses. Here Murata's color and compositional techniques appear analog, resembling Bacon's blurs, where, Deleuze argues, figure and abstraction emerge through color, destroying form and creating a new, dynamic "monstration" or, synthesized motif as the seed and rhythm of a world to come. ${ }^{47}$ At the same time, Murata's careful sculpting of polychromatic noise into tightly controlled color animations is far from an unstable becoming. Rather, he enforces a structure back into the wildness of the color affects that he initially unleashed in the composition. The artist's mastery over the medium, with its invisible yet pervasive attempts to shape content is rearticulated 
here through a balanced play between control and failure. In sum, glitch aesthetics and datamoshing in particular, with its still eccentric edge, provides us with a visual language derivative of, and suited for, the specific contours of the technologically mediated present. And this brings us to the third and final framework: historical contingency.

\section{HISTORICAL APPROACHES TO STYLE}

What is edgy and new in one paradigm is cliché in another. One way to understand the transitions between an emergent cultural style and the obsolescence of an older one is to turn to periodization claims. In 1915, the German historian Heinrich Wölfflin provided an especially compelling account of the differences in the shift from the High Renaissance to the baroque in the sixteenth and seventeenth centuries. It is "for the sake of simplicity," he argued, that "we must speak of the sixteenth and seventeenth centuries as units of style," even though they do not signify a homogeneous mode of production and have features that appeared prior to $1600 . .^{48}$ Wölfflin looked for general, overarching trends in a historical period, rather than narrow or directly causal chronologies. ${ }^{49}$ An era's style is also indisputably linked to technology, material culture, and the ways in which creative practitioners adopt, or fail to adopt innovations. The popular use of saturated, synthetic colors in the 1960s, for example, unified an era through similar color choices in interior design, fashion garments, light shows, and posters. Scholars of historical periods from Wölfflin through Jonathan Crary have gone to great lengths to articulate how contrasting and contradictory forces coexist in a given culture's zeitgeist. To speak of a period style is thus a generalization, but also, largely accurate.

Wölfflin's analysis begins with an examination of the linear versus the painterly, followed by a consideration of distinctions between plane versus recession; closed versus open form; multiplicity versus unity; and absolute versus relative clarity. I focus here only on his first set of terms: the linear versus the painterly, proposed as synonyms for the digital and the analog, respectively. For Wölfflin, the linear is in line with the High Renaissance's striving to achieve an image of perfection. In such an image, all of the figures and forms will be clearly outlined, made distinct from each other, and clearly illuminated for the viewer..$^{50}$ In contrast, the painterly style, he proposes, conveys a sense of indeterminacy, accomplished through the play of color, light, and shadow, illustrated by a Giorgione or a Titian. ${ }^{51}$

The comparison between the linear and the painterly also reinforces age-old debates between disegno and colore, where disegno (the origin of the word "design") implies the use of clear line and a rational, formal, structure, and as such, is associated with honesty and moral rectitude. In contrast, colore is associated with luscious brush work, the instinctual and primitive, and all those "Other" things tied up in Western culture's chromophobia. ${ }^{52}$ Understood through Wölfflin's pairing, disegno is analogous to the linear, with its canvas structured by lines used to create 
TABLE 3. The Analog and Digital as Precursors to Modern Style

The "Painterly" Baroque Style (17th century)

- Non-planar approach to painting; objects may be shown from angles or slightly askew.

- Sensual forms, implicitly lush and celebratory of curves and contours.

- Unity is accomplished through subordination of all elements into a single theme or motif. Elements thus appear integrated and inseparable.

- Flow and movement created through color and the play of light (arguably connected to the origins of animation).

- Emphasis on becoming versus being.

- Phenomenal representation of things as they appear.

Examples:

- Sandro Botticelli's “impetuous verve and animation";

- Gian Lorenzo Bernini’s "turbulent figures";

- Jacob van Ruysdael's "grave style" of undulating outlines, "compacting the mass of foliage" into a "ponderous," somber whole.
The "Linear" High Renaissance Style

(16th century)

- Straightforward, planar approach and grid-like representation of objects, making them appear flat and parallel to the picture frame.

- Implicit reliance on clear and well-defined lines to create volume.

- Unity is accomplished through rationalized balance of component parts.

- Color is structured through a composition's more dominant lines.

- Image appears static, studied, and balanced.

- Objects appear separate and detachable from the whole, though contained within the frame.

- Political and ideological correspondence with Western logos, claims to truth, knowledge, and the empirical sciences of the Enlightenment.

- Emphasis on being versus becoming.

- Claims to absolute clarity and the representation of things as they "actually are."

Examples:

- Lorenzo di Credi’s "deliberate modeling and repose";

- Gerard ter Borchs's "peaceful, delicate little pictures" (Wölfflin argues his style is more linear than Bernini's).

- Meindert Hobbema's freer "bounding lines" that rise "airily" and gracefully in space

NOTE: Quotations are all from Heinrich Wölfflin's Principles of Art History.

a clear visual path to guide the eye. Elements and figures maintain distinction from each other, akin to the discrete logic of the digital. The painterly, in contrast, prioritizes color (colore) as the means and method of expressing form. Paint is handled loosely, forms are less defined, lines are less discrete, and edges tend to be blurred and less readily apparent than in the linear method. The more "limitless" and "colossal" baroque aesthetic of colore aspires to dissolve into the sublimity of the infinite, creating a feeling of opening and "play rather than proof, illusion rather than reality, effects rather than resemblance." ${ }_{53}$ Thus the painterly qualities of the baroque are affiliated with the mixing and mystique of analog colorism, and, let us now suppose, the melting chunks of pixels in datamoshing. 
On the one hand, then, datamoshing tends to the painterly. It prioritizes color (colore) with less defined shapes, layers, and blurs. It also finds affiliation with the baroque aesthetic in that it draws on ambiguous techniques to articulate changing relationships between the individual and the world. In this case, it is not an aspiration to the divine, but instead, a grasp to make sense of the many obfuscations engendered through high-tech. We can further identify stylistic affiliations between the baroque and datamoshing if we recall that the former sought to render new relationships between the individual and the world, in the same way that I have suggested glitch art and datamoshing struggle to articulate the challenges indigenous to a new world of rapidly changing technology. Andrew Benson's Sparkle Face Test (2011) and Status Update, 2 AM (2011) also illustrate this tension. In both works, we witness an unresolved struggle between man and machine. In Status Update 2 AM, the performer (Benson) is immersed in a hurried technosound space, overwhelmed, but keeping up by drawing colored lines in synch with the frantic pace, ostensibly working past 2 A.M., even though this human, relative to the machine, seems doomed to fail by virtue of being too slow and too human.

On the other hand, datamoshing falls under the linear, not deliberately, but by default. Insofar as it and many other forms of glitch art are produced through digital technology, where the conditions of possibility for creative production are always already limited to $8 \times 8$ macroblocks of pixels, numerically confined to unique locations on an invisible grid. As John Belton puts it, "the digital image is too ordered and too rational-and not random enough. In our experience of it, it lacks ... 'being,'." 54 This may be so but what other option do we have for giving face to the human struggles in a digital culture? Additionally, one obvious difference between the baroque and datamoshing is that the former captured the angst of change while simultaneously remaining open to new aspirations and sublimity. In contrast, glitch art and datamoshing are from the start cynical of techno-optimism and faith in any expansive, transcendental register. This is amplified through the anxieties created by our dwindling control over our socioeconomic and political situations, making life in digital culture seem less connected to the limitlessness of the baroque era, and much more in tune with the "black box" framing discussed at the end of chapter 2 . In sum, datamoshing accurately stages the particular anxieties and tensions of life in the twenty-first century. At the same time, and by way of the above, it perpetuates cultural fantasies of mastery and control. That is, it plays according to the rules of computational order, and its negotiated diagram is thus more code than sensuous spontaneity. And yet, again, this contradictory mix of breakdown and control in the genre is, precisely, an accurate rendering of our real relations to the high-tech world we live in.

\section{Glitch Out}

One could argue that glitch art succeeds in making the matter of digital technology immanent, by conflating content and its mode of expression. But it is also true 
that we always fail to attain this goal (if it is one). Each artist must master his or her data set and then reorder it in a cool and controlled fashion. It also goes without saying that the vast majority of cutting-edge effects make their way to the center stage of commercial media and as they do, lose whatever political edge they may have once had. The following concluding anecdote illustrates this point.

One morning in March 2009, less than two months before the opening of Paul B. Davis's second solo show at the Seventeen Gallery in London (in which Compression Study \#1 was to be included), he woke up to find a flood of emails, "telling me to look at some video on YouTube." On his computer a few seconds later, Davis "saw Kanye West strutting around in a field of digital glitches that looked exactly like my work. It fucked my show up . . . the very language I was using to critique pop content from the outside was now itself a mainstream cultural reference." 55 The field he alludes to is the mise-en-scène for Kanye West's 2009 Welcome to Heartbreak music video, directed by Nabil Elderkin. Elderkin's video displays West strutting on stage with Kid Cudi, immersed in chromatic glitch artifacts. In the video, colors dance across the visual field, echoing the psychological heartbreak denoted in the lyrics. Given the music video's widespread commercial success, Welcome to Heartbreak illustrates how the proliferation of datamoshing and glitch styles have little to do with challenging habits of visual consumption or sustained critique, but instead with the effective control and engineering of mass media entertainment.

Kim Cascone refers to this neutralization of an experimental edge as a new style paradigm of "competitive consumption" indigenous to a "post-digital" age. ${ }^{56}$ Glitch and datamoshing's fate as a quickly appropriated mainstream special effect may then have more to do with recognizing this genre's failure as de facto in the way things work today; rapidly moving from so-called cutting-edge "innovation" to obsolescence as mainstream cliché. The cycles move so fast, one hardly has time to notice them. The appropriation of DIY effects into digestible forms of mass media is ubiquitous, intensified by the lightning speed of the internet and instantaneous digital processing. If we can slow down to take a closer look, as this chapter has done, we can catch a glimpse of the way in which these highly stylized digital artifacts have taken a thin slice of the process and temporarily abstracted it from clear channels of commerce and communication, giving us just enough time to catch it on the rebound and decode its broader value for understanding life as it is actually lived; strenuously and in eternal strife.

In Part III (chapters 6 and 7) and in the Postscript, I move away from an analysis of screen-based error to address phenomena in the culture and the environment that are failure-ridden as a result of high-tech industries. The material fallout resulting from the overproduction of so-called fast and efficient computational media has engendered inconceivable quantities of trash and e-waste, which, like noise and glitch, are unwanted but nonetheless necessary to consider if we wish to gain deeper insight into ourselves and likely futures. 
